\title{
Fintech e inclusión financiera: los casos de México, Chile y Perú*
}

Fintech and financial inclusion: the cases of Mexico, Chile, and Peru

\author{
Ignacio Esteban Carballo \\ Magíster en Inclusión Financiera y Microfinanzas, Universidad Católica Argentina, \\ Buenos Aires-Argentina, nachocarballo4@hotmail.com
}

Facundo Dalle-Nogare

Licenciado en Ciencias Económicas, Universidad Católica Argentina, Buenos Aires-Argentina, dallefacu@hotmail.com

\begin{abstract}
Cómo citar / How to cite
Carballo, I. E., \& Dalle-Nogare, F. (2019). Fintech e inclusión financiera: los casos de México, Chile y Perú. Revista CEA, 5(10), 11-34. https://doi.org/10.22430/24223182.1441
\end{abstract}

Recibido: 15 de febrero de 2019

Aceptado: 8 de mayo de 2019

\section{Resumen}

En este trabajo se propone abordar la temática de las tecnologías financieras, Fintech, y la relevancia que posee en contextos de fomento a la inclusión financiera. Para ello, se realizó una revisión de la literatura, partiendo de los principales conceptos que definen estas dos categorías, rescatando la mención de las microfinanzas como antecesora a la inclusión financiera. Específicamente, se estudian los casos de Chile, México y Perú en su evolución reciente (previa a 2018) concluyendo que, si bien los tres casos han realizado importantes avances, los esquemas institucionales en cada uno son muy diferentes, resultando en oportunidades y desafíos disímiles para cada uno. Se concluye afirmando que los canales que determinan la relación entre las Fintech y la inclusión financiera son extensos y heterogéneos. Es necesario impulsar políticas que comprometan de manera sostenible esfuerzos de los sectores públicos y privados para mantener vigorosos los procesos de inclusión financiera adaptados a las nuevas tecnologías. De manera general, este trabajo arroja luz al proceso de digitalización que atraviesan las finanzas y denota la heterogeneidad propia del mismo.

Palabras clave: inclusión financiera, Fintech, tecnologías financieras, economías emergentes.

\section{Abstract}

This work addresses financial technology (Fintech) and its relevance in contexts of promotion of financial inclusion. For that purpose, a literature review was conducted using the most important

\footnotetext{
* Este artículo se deriva del proyecto titulado "Fintech e Inclusión Financiera: los casos de México, Chile y Perú" y ha sido
} financiado con recursos propios. 
terms that define those two fields, and microfinance was highlighted as the predecessor of financial inclusion. More specifically, the recent evolution (before 2018) of the cases of Chile, Mexico, and Peru was studied to conclude that, although important progress has been made in the three countries, the institutional framework is very different in each one of them, which results in dissimilar opportunities and challenges in every context. It is concluded that the channels that determine the relationship between Fintech and financial inclusion are wide ranging and heterogeneous. Policies that engage sustainable efforts by the public and private sector should be promoted to support financial inclusion processes adapted to new technologies. Overall, this work sheds light on the digitalization processes financial services are undergoing as well as their heterogeneity.

Keywords: Financial inclusion, Fintech, financial technology, emerging economy.

\section{INTRODUCCIÓN}

Las Fintech (contracción de 'Finanzas' y 'Tecnología') son una afamada rama dentro de la innovación que propone oportunidades y desafíos a la oferta, demanda y regulación de los servicios financieros. Sin embargo, términos como Fintech (o BigTech, RegTech, InsurTech, AgTech, entre tantas otras variantes) son conceptos del marketing y carecen actualmente de contenido preciso o estandarizado (Rojas, 2016).

Por su parte, la inclusión financiera es un concepto amplio, polisémico y multidimensional, que también se encuentra en constante evolución, construcción y debate. Su multidimensionalidad parte de la necesidad obligada de contemplar diversos elementos y variables para alcanzar sus objetivos. Es por lo tanto un concepto inacabado cuyo desarrollo puede ser analizado e impulsado desde diferentes ópticas (Carballo, 2018).

En reconocimiento a la importancia de las nuevas tecnologías y los modelos empresariales innovadores para ampliar la inclusión financiera, el G20 elaboró el documento Principles for Innovative Financial Inclusion en 2010, mediante el cual se impulsaron medidas de política y esfuerzos nacionales para facilitar la innovación en los servicios financieros. Posteriormente, con el informe G20 High Level Principles for Digital Financial Inclusion, creado por el Global Standard-Setting Bodies and Financial Inclusion -GPFI- en 2016, se planteó el objetivo de canalizar acciones gubernamentales para impulsar la inclusión financiera a través de las tecnologías digitales y también proporcionar una base para los planes de acción (G20 Research Group, 2010), (Global StandardSetting Bodies and Financial Inclusion, 2016; (Arner, Barberis \& Buckey 2016).

En The 2016 Maya Declaration Report (2016) se mostraba cómo los servicios financieros digitales figuraban entre las tres áreas temáticas principales de los objetivos y compromisos asumidos por los países miembros de la Alliance for Financial Inclusion - AFI — en África Subsahariana y Asia.

De igual modo, cada vez más los gobiernos nacionales y las instituciones se comprometen a digitalizar sus entidades financieras y los grandes flujos de pagos. Esto refleja cómo las Fintech cobraron un papel significativo en la inclusión financiera, que está siendo apoyado por estándares globales y organismos clave de inclusión financiera. 
En el presente trabajo, se analiza el desarrollo reciente de la inclusión financiera y las tecnologías financieras en tres economías seleccionadas. Específicamente, se busca a través de un método descriptivo y con base en la literatura, contrastar la composición institucional del sector Fintech y el avance de la inclusión financiera en cada país, junto con las distintas iniciativas adoptadas.

El objetivo de este artículo, se esgrime sobre la necesidad de comprender la heterogeneidad propia de cada economía, como instancia previa para analizar los desafíos y oportunidades teóricas (esto es, no evaluadas empíricamente) que presentan las Fintech a la inclusión financiera. Para comprender esta problemática, se consideró el contexto de tres economías de América Latina en su evolución reciente (previa a 2018) que, si bien culturalmente presentan similitudes, son muy variadas en cuanto al contexto institucional y la intervención estatal. En esta línea, este estudio tiene como objetivos específicos analizar las Fintech en cuanto a su relación con la inclusión financiera en cada uno de estos contextos. Adicionalmente, dependiendo la estructura institucional en cada caso, poder ver en concreto cuáles han sido los tipos de Fintech a los que mayor protagonismo se les ha dado y la evolución del fenómeno de inclusión financiera.

El artículo se estructura del siguiente modo. A continuación, en la segunda sección, se presentan definiciones conceptuales como marco teórico. Seguidamente, en la sección tres, se expone la metodología. Posteriormente, en el cuarto apartado, se llevará a cabo el análisis de tres casos de estudio, previo a realizar un análisis comparado en la quinta sección. Finalmente, se exponen las reflexiones finales a manera de conclusión.

La intención será dar lugar a la reflexión sobre la importancia que tienen las Fintech en la actualidad y estudiar distintos caminos a adoptar para fomentar su desarrollo conjuntamente a un proceso de fomento a la inclusión financiera.

Por último, es necesario remarcar la principal limitación de este estudio: el presente trabajo se circunscribirá exclusivamente a realizar un análisis descriptivo de dos fenómenos multidimensionales y en constante evolución para tan solo tres economías. Producto de ello, los resultados o implicancias de este artículo no son extrapolables a otras economías de América Latina y pueden en el futuro, producto del dinamismo en este sector, dejar de serlo para las aquí analizadas.

\section{MARCO TEÓRICO}

Como se mencionó, la inclusión financiera es un concepto amplio, polisémico y multidimensional que se encuentra en constante evolución, construcción y debate. Su multidimensionalidad parte de la necesidad obligada de contemplar diversos elementos y variables para alcanzar sus objetivos.

A grandes rasgos, se puede afirmar que la inclusión financiera implica la creación, promoción y regulación de un ambiente financiero accesible, asequible y seguro para el conjunto de la sociedad. Tiene como fin promover el bienestar económico y la inclusión social a través de la oferta de servicios y productos financieros diseñados para satisfacer las necesidades de distintos sectores de la población (Carballo, 2018). 
Es por lo tanto un concepto inacabado, cuyo desarrollo puede ser analizado desde distintas ópticas. Si bien la inclusión financiera ha ingresado paulatinamente en la agenda política internacional, el camino transitado por la misma posee un largo recorrido.

A fines del año 2015, los ocho Objetivos de Desarrollo del Milenio -ODM- puestos en marcha en el año 2000 llegaron a su fin. En aquella Asamblea General de las Naciones Unidas existió un acuerdo común sobre la necesidad de seguir trabajando en varios aspectos contemplados en los ODM, como son la pobreza, las diferencias de género y la desigualdad (Aguilar, 2016).

Consecuentemente, como sucesora de las "Metas del Milenio», se declaró la nueva Agenda 2030 para el desarrollo sostenible de las Naciones Unidas (ODS). Una característica a señalar es que la misma posicionó la inclusión financiera en un lugar prioritario, mencionándola en cinco de sus 17 nuevos objetivos. En este sentido, actualmente el debate se encuentra cerrado y la inclusión financiera es reconocida a nivel global como un factor fundamental para el desarrollo (The Economist Intelligence, 2015).

Sin embargo, el camino transitado por la inclusión financiera fue extenso y no nace con los ODS. Algunos hitos que la ubicaron en la agenda pública sucedieron en el año 2009, cuando el Grupo de los Veinte (G-20) estableció el tema como uno de sus ejes en la Cumbre de Pittsburgh, en el año 2011. Allí la Declaración Maya desarrolló una serie de compromisos orientados a incrementar el acceso a los servicios financieros formales a personas sin vinculación al sistema financiero, o el año 2013 cuando el Banco Mundial inicia su publicación del Global Financial Development Report con el objeto de estudiar el papel del Estado en las finanzas, a raíz de la crisis financiera global. Se debe señalar que el creciente interés es consecuencia también de una mejor comprensión al respecto de la importancia de la inclusión financiera para el desarrollo económico y social.

Con una mayor antelación histórica, las microfinanzas que nacen en Bangladesh y partes de América Latina a mediados de 1970 (Lacalle-Calderón, 2008), han sabido constituirse como una herramienta fundamental para reducir las disparidades de acceso a servicios financieros en personas en situación de pobreza excluidas por el sistema financiero tradicional. El principal motivo que ha excluido a los pobres del sistema financiero formal es una errónea consideración sobre su capacidad para ahorrar, pagar un crédito o iniciar un emprendimiento productivo.

Las microfinanzas nacen, entonces, como una innovación metodológica para ofrecer distintos servicios financieros a la población en situación de pobreza o sin colateral. Casi medio siglo de evolución y desarrollo ha llevado a que dejaran importantes lecciones respecto a cómo generar una efectiva inclusión financiera con foco en los más vulnerables.

Las microfinanzas como instrumento han tenido el claro objetivo de luchar contra la pobreza a partir del desarrollo de mecanismos que permitan dar lugar a la provisión de crédito y el ahorro a personas de bajo poder adquisitivo. El acceso al crédito es solo una de las posibilidades que comprende la actividad microfinanciera, ya que son varias las herramientas de las que dispone para acceder a productos financieros, como lo son, por ejemplo, los depósitos bancarios, los medios de transferencia o los seguros (Camacho-Beas, 2010; Orozco-Gutierrez, 2019). 
Su importancia radica en focalizar la atención sobre los individuos excluidos del sistema bancario formal, promoviendo el acceso a determinados productos y servicios bancarios, y buscando siempre satisfacer las necesidades financieras de los más desfavorecidos. Es esta la definición que denota la relación entre las microfinanzas y la inclusión financiera (Vázquez, 2016).

Así, más allá del microcrédito inicial, hoy en día se disponen de otras herramientas para afrontar la exclusión financiera en la base de la pirámide, tales como productos de ahorro y sistemas de pago. Entre estas, la tecnología ha sido una herramienta que se ha venido desarrollando, y junto con ella, el alcance de los servicios financieros. Puede decirse que la tecnología está revolucionando el acceso y el uso de los servicios financieros, del mismo modo que el internet y los dispositivos móviles transformaron la forma de interactuar entre las personas (The Economist Intelligence Unit, 2018).

Las principales responsables detrás del desarrollo del tópico claramente han sido las instituciones microfinancieras. Este tipo de instituciones se han presentado como una verdadera revelación, eliminando la necesidad de avales o garantías patrimoniales y creando un sistema bancario sobre los cimientos de la confianza, la participación y la responsabilidad. A modo de mención, estas pueden distinguirse entre ONG, bancos comerciales y entidades gubernamentales (Lacalle-Calderón, 2008).

Por supuesto, el entorno macroeconómico en el que estas instituciones se desarrollan juega un papel preponderante para explicar las diferencias en su desempeño y su composición. En esta línea, no hay duda de que el campo tecnológico ha sido el que más se ha desarrollado durante las últimas décadas, y esto se profundiza aún más cuando se trata de tecnología relacionada a las telecomunicaciones. Desde la década de 1970, con el comienzo de la llamada «Era Digital», el enorme avance tecnológico ha provocado un giro de 360 grados al momento de producir bienes y prestar servicios (Varas, 2017).

En el sector financiero, históricamente los bancos han sido los pioneros de toda innovación que se ha ido presentando. La aparición de las tarjetas de crédito en la década de 1950 y la posterior invención de los cajeros automáticos, tan solo una década después, cambiaron totalmente las interacciones financieras. Sin embargo, hoy en día gracias a las nuevas empresas de Tecnologías Financieras (Fintech), la innovación por parte de los bancos parece haber pasado a un segundo plano (Chishti, Barberis, \& Vidal, 2017).

Específicamente, la palabra Fintech apareció por primera vez en los medios en 2005, recién logrando virilizarse a gran escala en el año 2014 (Bosch-Liarte \& Bosch-Liarte, 2016). Del mismo modo, empresas Fintech son aquellas encargadas de ofrecer, mediante plataformas tecnológicas, una variada gama de productos o servicios financieros a los consumidores de forma efectiva y accesible (Silva \& Ramos 2017).

En cuanto al momento de su creación, si bien las empresas Fintech no son nuevas (Bloomberg fue creada en 1981 y Paypal a fines de la década de 1990), la cantidad de emprendimientos en este segmento aumentó exponencialmente luego de la crisis mundial del año 2008 (Torres, 2017).

Según Torres (2017), las empresas Fintech pueden clasificarse del siguiente modo:

- Fintech de financiación

- Fintech de asesoramiento 
- $\quad$ Fintech de transferencias, cobros y pagos e-commerce

- Fintech de contabilidad distribuida o blockchain

- Fintech de criptodivisas

El fenómeno de inclusión financiera y las microfinanzas, lejos de ser una excepción, también se encuentran inmersos en la «revolución digital» que el imparable avance tecnológico ha estado impulsando a lo largo de las últimas décadas. El potencial es enorme y el alcance innegable, basta mencionar que hoy en día existen más conexiones móviles que habitantes en el mundo. A su vez, la tecnología apunta a ser el camino más viable para hacer frente a cuestiones de alcance y la entrega de servicios en zonas periféricas (Ontiveros, Enríquez, \& López, 2014).

La mayor oportunidad radica en los países emergentes que cuentan con un índice de bancarización generalmente inferior a la penetración de las tecnologías móviles. De acuerdo con la consultora Mckinsey, en 2014 casi el $80 \%$ de los adultos en economías emergentes tenían suscripciones móviles, en comparación con el 55 \% que tenían una cuenta financiera. Además, el dinero en efectivo figura en el $90 \%$ de las transacciones, lo cual implica un grave inconveniente para las instituciones de microfinanzas. Por un lado, porque quedan sujetas a mayores costes operativos que implica el manejo de efectivo, además porque tienen una menor capacidad de «lectura», o recopilación de información por parte de los posibles prestatarios (Manyika, Lund, Singer, White, \& Berry, 2016; Bermeo-Giraldo, Álvarez-Agudelo, Ospina-Rúa, Acevedo-Correa \& Montoya-Restrepo, 2019).

Ahora bien, las grandes ventajas traen consigo grandes desafíos relacionados con las complejidades aparentadas al funcionamiento y a la confianza de los usuarios. Algunos autores opinan que más allá de las bondades de las Fintech, si perdieran la confianza, si dejaran de ser seguras o bien si dejaran de respetar la ley, no se dudaría en abandonarlas.

Como queda claro, ahondar en la relación entre inclusión financiera y Fintech se transforma en una temática de total vigencia y actualidad. Pero también se transforma en una temática inmersa en un marco teórico que está aún en plena gestación y que requiere esfuerzos de análisis y conceptualización particulares. En este trabajo se intentará denotar dicha complejidad a la vez de realizar un análisis reciente de los casos de estudio.

\section{METODOLOGÍA}

Este documento es el resultado de una revisión crítica de la literatura sobre un tema en particular: inclusión financiera y Fintech en México, Chile y Perú. Por eso, se realizará desde un enfoque exploratorio-descriptivo, partiendo sobre la base de la escasa información disponible sobre el tema de análisis.

Las cifras y métricas referidas al sector estatal se obtuvieron en gran parte de ensayos o informes expuestos por ministerios, asociaciones y bancos públicos; mientras que cuando se trata del sector privado, los datos surgieron mayoritariamente de ensayos de organizaciones, siendo el Consultative Group to Assist the Poor - CGAP - y el Banco Interamericano de Desarrollo - BID-, los máximos referentes. 
En cuanto al análisis de casos, para cada país va a respetarse una misma estructura. En primer lugar, se menciona brevemente la evolución reciente de la inclusión financiera y las Fintech dentro de cada economía. En segundo lugar, se describen las instituciones que rigen dentro del sector junto con las principales empresas Fintech. Por último, se abordan la evolución en términos de inclusión financiera. Dada la acotada evidencia empírica que existe actualmente en esta temática, se seleccionaron entre 5 y 6 variables (dependiendo la economía) para denotar la incorporación de las Fintech en cada país y la consecuente evolución de la inclusión financiera. La idea detrás de esto fue poder estandarizar los resultados a través de las mismas variables que se corresponden a los años 2011, 2014 y 2017, todas estas propias de la base de datos del Banco Mundial.

En resumen, se analizará (a) el contexto institucional, (b) las Fintech en el territorio, y (c) la evolución de la inclusión financiera. Siendo este un análisis descriptivo de la situación actual de las dimensiones de análisis, esta metodología permitirá tener una lectura de tres casos de estudio que, como se puede apreciar, presentan similitudes, pero también amplias diferencias.

\section{RESULTADOS}

Un comportamiento importante que debe destacarse a priori sobre las Fintech y su papel en América Latina es que muestran una clara tendencia a servir segmentos de la población que hasta ahora no estaban cubiertos por el sistema financiero tradicional $y$, tal vez marginalmente, por las microfinanzas. Esto resulta sumamente positivo en una región marcada por la desigualdad y la exclusión del sistema financiero.

En una encuesta realizada por el BID en el año 2017, se obtuvo como resultado que sobre un total de 393 jóvenes empresas Fintech en Latinoamérica, aproximadamente el 40 \% de estas afirmaba que su misión era servir a clientes que permanecían excluidos o subatendidos por el sector de los servicios financieros tradicionales fomentando así la inclusión financiera. A su vez, hay dos modalidades que representan poco más de la mitad de la totalidad de las categorías Fintech en la región: Fintech de financiación $(25,6 \%)$ y Fintech de transferencias, cobros y pagos e-commerce (25,2%) (Banco Interamericano de Desarrollo, 2017).

Con respecto a los países de estudio, Chile contaba a inicios del 2017 con el 72 \% de su población con una cuenta en una institución financiera; mientras que México y Perú manejaban cifras cercanas al $39 \%$ y $44 \%$ respectivamente. La urgencia y necesidad de desarrollo en estos últimos dos casos quedaría reflejada con la sanción de una Estrategia Nacional de Inclusión Financiera (Trivelli y Caballero, 2018).

\section{El caso de México}

México es un país particular para estudiar la inclusión financiera dentro de la región. Su evolución, tipos de instituciones y servicios ofrecidos a los estratos sociales más bajos denota un claro compromiso en promover el acceso a los servicios financieros formales (Villacorta \& Reyes, 2012).

De acuerdo con el World Bank Group (2017), actualmente solo el 39 \% de los mexicanos cuenta con acceso a servicios financieros formales. Según la Encuesta Nacional sobre la Penetración y 
Conocimiento de Servicios Financieros, realizada por la Secretaría de Hacienda en el año 2006, este porcentaje descendía a un $15 \%$.

Para entender cómo se ha logrado casi triplicar el acceso a servicios financieros en poco más de una década, es importante mencionar todos los promotores de esto, dentro de los cuales las Fintech cumplen un rol protagónico (Raccanello y Herrera, 2014).

\section{Contexto institucional}

Entrado el año 2009 es cuando en México se comienza a hablar de inclusión financiera como concepto de política pública, y en 2011 se logra definirla como "el acceso y uso de servicios financieros bajo una regulación apropiada que garantice esquemas de protección al consumidor y promueva la educación financiera para mejorar las capacidades financieras de todos los segmentos de la población". (Raccanello y Herrera, 2014, p. 18).

De 2011 a la actualidad, puede decirse que el Gobierno mexicano ha canalizado su apoyo mediante tres acontecimientos marcados: (i) la creación del Consejo Nacional de Inclusión Financiera (CONAIF) en el año 2011, con el objetivo de llevar a cabo una acción más coordinada de las autoridades financieras; (ii) la implementación de programas y acciones para fortalecer la inclusión financiera, tales como la bancarización de los beneficiarios de los programas sociales; y (iii) el esfuerzo en la obtención de más información valiosa (mediante reportes y encuestas nacionales) que permita guiar la acción gubernamental y difundir el estado de IF en el país (CONAIF, 2016).

Ahora bien, para ese entonces no se había realizado mención alguna sobre las Fintech como herramienta clave para combatir la exclusión financiera. Esto recién ocurre para el 2016, año en el que la CONAIF sanciona la Política Nacional de Inclusión Financiera (PNIF), y considera el aprovechamiento de las innovaciones tecnológicas como un eje central para la IF (Silva \& Ramos, 2017).

En lo que respecta a la regulación, recién para inicios del 2018, el Estado mexicano se encargó de sancionar una ley sobre las Fintech: la «Ley para regular las Instituciones de Tecnología Financiera». Previa a esta, no existía una ley específica que regulara esta industria, por lo que muchas de las empresas del sector habían estado operando en áreas grises de las ya existentes. Cabe mencionar que es el primer caso que existe en la región (Ocampo, 2017).

\section{Fintech en territorio}

Al tratase de una economía en vías de desarrollo, las Fintech que mayoritariamente abundan en su territorio son aquellas que buscan promover una mayor inclusión financiera y reducir la pobreza.

Como señaló BID economics México, la conectividad digital tiene que entenderse como la puerta para la integración de México en la economía global, logrando una reorganización eficiente de los procesos de producción y consumo. En cuanto a las categorías que mayor presencia tiene en el territorio, los primeros lugares están ocupados por Fintech de financiación (59) y Fintech de pagos (50); mientras que el podio lo completan las Fintech de asesoramiento (22) (Banco Interamericano de Desarrollo, 2017). 
Dentro del desarrollo de la inclusión financiera, las empresas Fintech han sido las protagonistas, en cierta medida por la falta de iniciativas concretas por parte del Estado (más allá de adoptar una ENIF genérica). El apoyo por parte de capitales privado se ha hecho notar de modo que las empresas Fintech en México, que aumentaron en un 50 \% en cantidad entre 2016 y 2017, recibieron una inversión superior a los USD 89 millones entre diciembre 2015 y enero 2017 (Ocampo, 2017).

\section{Evolución de la inclusión financiera}

A continuación, se presentarán una serie de variables que se consideran apropiadas para suponer los resultados que han tenido las Fintech en México durante los últimos años. Es importante remarcar que no se trata de una relación lineal, sino que hay muchas variables que inciden sobre estas y las Fintech podrían estar influyendo al menos teóricamente en el grado de desarrollo. Ver Figura 1:

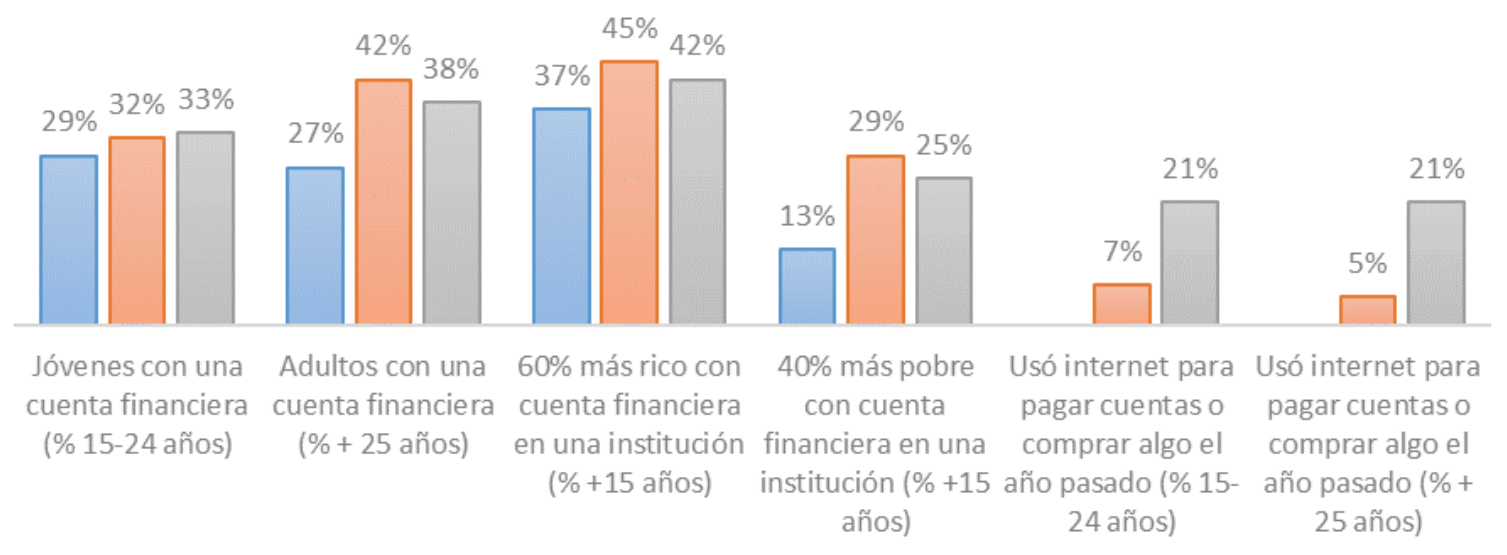

$\square 2011 \square 2014 \square 2017$

Figura 1. Evolución de los servicios financieros en México

Figure 1. Evolution of financial services in Mexico

Fuente: elaboración propia con datos de (Demirguc-Kunt, Klapper, Singer, Ansar, \& Hess, 2018).

Como se observa en la Figura 1, los dos primeros conjuntos de columnas demuestran un incremento en la tenencia de cuentas financieras, tanto en personas menores como mayores de 25 años, con un mayor incremento en este último grupo. Seguramente la elevada adopción de tarjetas de débito por parte de la población adulta durante los últimos años sea en gran medida el motivo: entre 2012 y 2015 el porcentaje de adultos con tarjeta de débito se incrementó en 7 puntos porcentuales, pasando de $35 \%$ a 42 \% (Comisión Nacional Bancaria y de Valores, 2017).

En segundo lugar, en lo que respecta a la clase social, puede apreciarse que el incremento es considerablemente superior para aquellas personas con menores ingresos. Detrás de esta diferencia se sitúa la ya mencionada PNIF, orientada en mayoría para toda aquella población sub-atendida y excluida (Comisión Nacional Bancaria y de Valores, 2017).

Por último, y considerando la totalidad de las series, una clara distinción generacional que es importante recalcar es que los individuos más jóvenes (15-24 años) tienen una mayor adhesión al uso 
del internet como medio de pago, mientras que las personas de mayor edad (+25 años) son los de una adhesión superior a las cuentas financieras. La relación natural e intrínseca que mantienen los jóvenes con la tecnología, sumada a su pérdida de confianza en el sistema financiero tradicional seguramente explican en parte esta diferencia (Dib, Ramírez \& Alvarado, 2017).

\section{El caso de Chile}

A diferencia de los casos de México y Perú (que se podrá apreciar más adelante), Chile presume de un alto grado de bancarización y un mercado financiero desarrollado, alcanzando, entre otras metas, el porcentaje más alto (74 \%) de personas con una cuenta en una institución financiera en América Latina hacia el año 2017 (Trivelli y Caballero, 2018).

En lo que respecta a la incursión de las Fintech, son varios los reportes que sugieren a Chile como uno de los países de la región en los que más desarrollo ha tenido dicho sector. Esto particularmente se debe a la gran presencia del financiamiento colectivo o crowdfunding. En el año 2016, el volumen de transacciones en Chile fue el segundo mayor (USD 97.8 millones) en la región, situándose únicamente por detrás de México (USD 334.5 millones) pero por encima de Brasil (USD 12.6 millones) (Ziegler, Reedy, Le, Zhang, Kroszner \& Garvey, 2017).

De todos modos, el rasgo distintivo más simbólico del escenario chileno es otro: la fuerte incidencia que históricamente ha tenido el Estado sobre la inclusión financiera, y más precisamente a través del Banco Estado; el único banco comercial estatal en Chile creado en el año 1953. Ahora, si bien desde la entidad sostienen haber promovido la inclusión financiera desde sus inicios, las medidas más disruptivas se han visto en las últimas décadas, tales como la creación del Programa de Microempresas de 1996 o bien el sistema CuentaRut de 2006.

\section{Contexto institucional}

A diferencia de México, que tuvo un punto de inflexión en su historia mediante la sanción de la Política Nacional de Inclusión Financiera (PNIF) en el año 2016, la performance chilena ha demostrado un compromiso histórico con la inclusión financiera a través de iniciativas más graduales y sostenidas en el tiempo. Como se dijo, estas han surgido propiamente desde el Estado (operando mediante el Banco Estado), siendo una clara diferencia con respecto a los otros casos de estudio. En la Figura 2 se listan las más importantes.

Cuando se habla de las principales medidas que se han adoptado en Chile para promover la inclusión financiera, las Fintech merecen ser mencionadas, puesto que las dos más importantes han requerido de una gran alianza con las tecnologías financieras para lograr su impacto: la Sociedad Caja Vecina y el Sistema de CuentaRut, las cuales serán descritas más adelante.

La importancia detrás de esto es que no actúan como sustitutos; son tecnologías complementarias que han sabido generar sinergias entre sí. La red de corresponsalías no solo incrementa de manera significativa el número de puntos de atención presencial en que puede usarse la CuentaRut, sino que también permite la utilización de las mismas en horarios que exceden al de las sucursales bancarias (Superintendencia de Bancos e Instituciones Financieras Chile, 2016). 
- Caja Vecina: El BancoEstado constituye la Sociedad de Servicios de Transacciones Caja Vecina S.A. para administrar la red asociada a la banca corresponsal de la entidad.

- Cuenta RUT: El BancoEstado lanza el producto de manera Universal para todo habitante en territorio chileno.

- Cumbre del G20: Chile se compromete a crear una instancia de coordinación de alto nivel para liderar la agenda nacional de inclusión financiera.

-Programa Chile Cuenta: se crea con el objetivo de promover el pago de los beneficios sociales estatales a través de una cuenta bancaria. Este entraría en vigencia recién en Junio del año siguiente, con una tasa de aceptación promedio del $53 \%$.

- Secretaría Técnica de IF: se crea con el objetivo de avanzar con el diseño de una Agenda Nacional de Inclusión Febrero Financiera.

2013

- Comisión Asesora para la IF (CAPIF): Mediante un decreto (N. 945) del Ministerio de Hacienda, se crea esta comisión con el objetivo de asesorar a la Presidenta en todo lo referente al diseño y gestión de la Estrategia Nacional de Inclusión Financiera.

Figura 2. Principales medidas orientadas al desarrollo de la IF en Chile

Figure 2. Timeline of most important measures to promote Fintech in Chile Fuente: elaboración propia con datos de (Demirguc-Kunt, Klapper, Singer, Ansar, \& Hess, 2018).

En lo que respecta a la regulación, Chile no dispone (al menos por ahora) de leyes o regulaciones específicas sobre el sector Fintech. De este modo, las entidades de este tipo han tenido que saber amoldarse y lidiar con los desafíos que implican operar dentro del marco regulatorio «tradicional». Las instituciones reguladoras suelen apoyarse en el argumento de que una innovación Fintech específica no forma parte de su área de supervisión, y por ende debe regirse mediante el marco regulatorio tradicional. Ahora, si bien este comportamiento no impide que se produzcan innovaciones, por lo general las regulaciones generales no suelen ser aptas para las Fintech (Furche, Madeira, Marcel \& Medel 2017). 


\section{Fintech en territorio}

Una vez más, distinguiéndose de los otros casos, en Chile históricamente las principales iniciativas Fintech han provenido del sector púbico: la CuentaRut (en cuanto a la utilización) y la Red Caja Vecina (en cuanto al acceso) (Ministerio de Desarrollo Social, 2015).

La CuentaRut entró en vigencia a partir del año 2006 y desde allí las cifras de alcance y cobertura a nivel nacional no han parado de crecer. Actualmente, se ha convertido en el principal medio de pago a nivel nacional, superando los 25 millones de transacciones hacia fines de 2012. Sin embargo, más importante aún es conocer la cantidad promedio de transacciones por cliente, ya que es a partir de este en el que verdaderamente se refleja la participación de la aplicación en el mercado. Dicho esto, entre 2006 y 2012 este valor fue más del cuádruple, pasando de 1.1 a 4.7 transacciones por cliente (Ministerio de Desarrollo Social, 2015).

La primera sucursal de la Red Caja Vecina fue inaugurada en el año 2005 en la localidad El Carmen, región de Biobío. Desde aquel entonces hasta la actualidad, se ha convertido en la red de corresponsalía más grande a nivel nacional, con presencia en todas las comunas. Tan solo para dimensionar la magnitud que ha implicado este proyecto por parte del BancoEstado (que hoy en día cuenta con más de 22 mil sucursales) se estima que tan solo aquella sucursal incidió en la IF, y social, de aproximadamente 14 mil personas (Maldonado et al, 2018).

\section{Evolución de la inclusión financiera}

Tras haber expuesto las principales iniciativas (en este caso estatales) Fintech, que ha habido en Chile durante los últimos años, se profundiza a continuación en los resultados en materia de inclusión financiera:

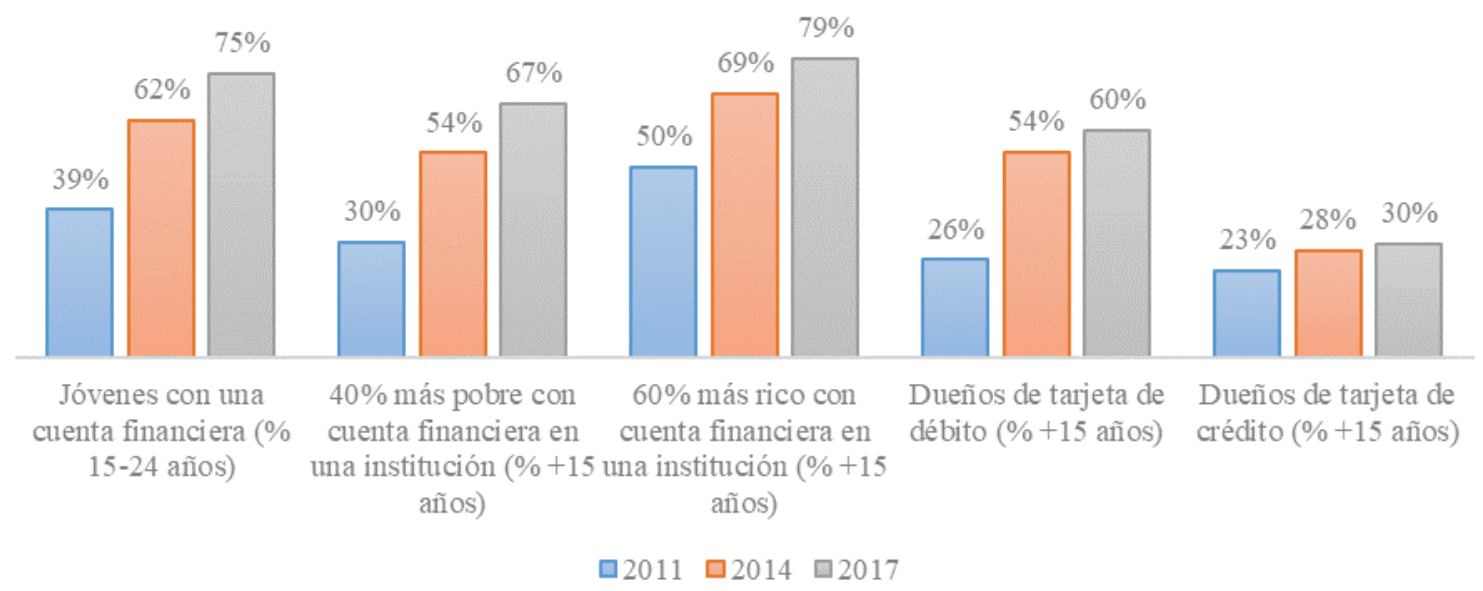

Figura 3. Evolución de los servicios financieros en Chile

Figure 3. Evolution of financial services in Chile.

Fuente: elaboración propia con datos de (Demirguc-Kunt, Klapper, Singer, Ansar, \& Hess, 2018). 
Como se extrae de la Figura 3, en el transcurso de este período de 6 años, todas las variables consideradas han presentado un crecimiento notable, si bien algunas partieron de una base menos desarrolladas que otras. El primer conjunto de columnas tiene como objetivo denotar el gran avance que ha habido durante estos últimos años en lo que respecta a la tenencia de cuentas financieras por parte de gente joven en Chile, creciendo 36 puntos porcentuales al pasar de $39 \%$ en 2011 al $75 \%$ para 2017. Este comportamiento va claramente en línea con el porcentaje de adhesión a la Tarjeta Cuenta Rut por parte de la gente joven (20-24 años) que previamente se había mencionado (Rojas, 2016).

Las siguientes series de columnas tratan de marcar otro tipo de categorización: la clase social. El comportamiento resulta ser similar en ambos casos, con una clara tendencia ascendente. La diferencia, sin embargo, radica en el grado de crecimiento, ya que mientras para el estrato de mayores ingresos el porcentaje entre el principio y fin del análisis aumenta un poco más de la mitad, en el caso de la gente con menores ingresos este se duplica. Seguramente las políticas del Banco Estado orientadas mayoritariamente a la clase social más baja (Cuenta Rut y Caja Vecina, entre otras) explica en gran parte este comportamiento (Banco Estado; 2015).

El último par de series de columna deben analizarse en conjunto. En este caso, las variables y/o elementos son distintos (tarjeta de débito versus tarjeta de crédito), pero el criterio es el mismo: porcentaje de posesión. La importancia de esta serie radica nuevamente en los grados de crecimiento; en el caso de las tarjetas de créditos, la posesión crece en menos de un tercio; mientras que para el de las tarjetas de débito este es mayor del doble.

Detrás de esto hay una única razón: Tarjeta Cuenta Rut. En la actualidad los registros marcan que hay más de 10,5 millones de personas con una de estas, y se estima que alrededor de 4 millones solo tienen esta tarjeta como único producto bancario (Vega, 2017).

\section{El caso de Perú}

En el transcurso de los últimos años las iniciativas, tanto de grupos del sector público como del sector privado, denotan el sentido de importancia que se le ha estado dando a la inclusión financiera en Perú.

Según un estudio realizado en 2018, a la par con Colombia, el país peruano ocupa el primer lugar en cuanto a políticas públicas y apoyo del Gobierno sobre la inclusión financiera. En lo que concierne a la demanda, el contexto es competitivo y abierto. Pueden mencionarse como características el hecho de que no existan topes en las tasas de interés de los préstamos o bien que se permitan operaciones de agentes no bancarios. En paralelo, el sector público y privado cooperan activamente entre sí para que promover el desarrollo de nuevos productos, como ha sido el caso del dinero electrónico (The Economist Intelligence Unit, 2018).

Es interesante denotar que el informe Global Microscope ha reconocido en reiteradas ocasiones este apoyo consolidado, al punto tal que ha distinguido a Perú en ocho ocasiones (2007-2015) como el país con el mejor entorno para la inclusión financiera, haciendo un especial hincapié en la participación estatal que se ve reflejada en la coordinación de los distintos agentes. 
Estos informes se han centrado principalmente en el análisis de su sector de microfinanzas, no así en la inclusión financiera propiamente dicha. Entre las áreas que han presentado una mejora sostenida pueden distinguirse las siguientes: a) Democratización de los servicios financieros; b) Fortalecimiento de las instituciones financieras; c) Mejora en protección al consumidor; d) Normativas para el uso del dinero electrónico (Zamalloa, Obando Peralta \& Rodríguez Cairo, 2016).

\section{Contexto Institucional}

Iniciado el siglo XXI, el crecimiento de la economía peruana puede entenderse como la principal causa que alentó una expansión interna de su sistema financiero. Entre otros eventos, puede resaltarse como motivo el crecimiento de la intermediación de las instituciones financiera, todas estas reguladas por la Superintendencia de Banca, Seguros y AFP (SBS).

La SBS se presenta entonces como la principal entidad reguladora, y así los indica la Ley General del Sistema Financiero y del Sistema de Seguros, desde el año 2004 (Aguilar, 2016).

Ahora bien, no solo desde el aspecto institucional Perú cuenta con un clima favorable para el desarrollo de las microfinanzas y fomento a la inclusión financiera, sino que ha ido adoptando paralelamente estrategias tecnológicas que también contribuyeron para que se convierta en uno de los países pioneros.

Hacia fines del año 2017 se registraron 48 empresas Fintech en Perú, con una mayoría operando dentro de la categoría Fintech de financiación. La aparición de estas ha sido disruptiva, inclusive ha encendido la alarma de la banca tradicional que ha visto cómo sus clientes poco a poco se han alejado, optando por las herramientas más amigables de las Fintech.

De todos modos, desde el mismo Lima Fintech Forum realizado en el 2017, se ha dejado claro que la intención no es que las Fintech y la banca tradicional compitan entre sí, sino que sean colaborativos entre sí y se consideren mutuamente como socios estratégicos (Asociación de Bancos del Perú, 2017).

En lo que respecta a la regulación sobre esta área, la misma SBS sentenció el año pasado que se ha estado gestando y evaluando una propuesta regulatoria integral para incorporar a las Fintech dentro del marco normativo.

\section{Fintech en territorio}

Al igual que en los casos previamente mencionados, las Fintech de financiación son las que mayor participación tienen en el país. En los países de América Latina, al menos una de cada cuatro Fintech opera como plataforma alternativa de financiación, y Perú lejos está de ser la excepción (Campos \& Huangal, 2017).

Si bien todavía sigue en discusión si las iniciativas Fintech van a trasformar el mercado financiero por la vía de la competencia o, más bien de la colaboración con las empresas del sistema financiero, es evidente que la gente las ha ido adoptando. Según la Encuesta Nacional de Demanda de Servicios Financieros y Nivel de Cultura Financiera en el Perú del año 2016, del 67 \% de personas que ahorran en Perú, solo el 17 \% lo hace en el sistema financiero tradicional (Varas, 2017). 


\section{Evolución de la inclusión financiera}

Así como se hizo previamente, van a tomarse en consideración una serie de variables representativas de las mejoras en materia de inclusión financiera que ha habido en el país desde 2011.

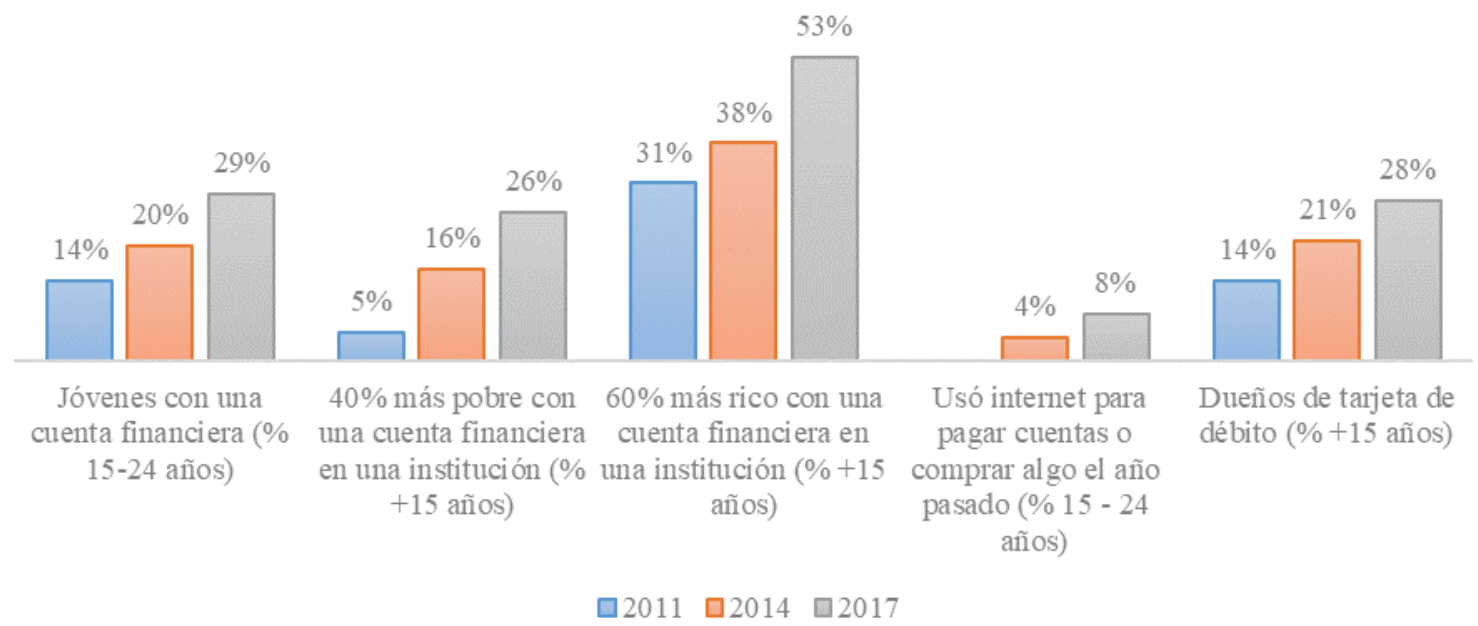

Figura 4. Evolución de los servicios financieros en Perú

Figure 4. Evolution of financial services in Peru

Fuente: elaboración propia con datos de (Demirguc-Kunt, Klapper, Singer, Ansar, \& Hess, 2018).

Como primer comentario genérico, al analizar la Figura 4, puede decirse que ha prevalecido una tendencia alcista en todas las variables consideradas, lo cual denota avances durante el tiempo de consideración en lo que respecta a inclusión financiera y una mayor adhesión en el uso de la tecnología. Casi en la totalidad de los indicadores, el porcentaje entre el primer año de consideración y el último se duplica, por lo que puede hablarse de un incremento notorio.

Los números resultan ser los más bajos de los tres países considerados, y hay una serie de motivos detrás de esto: según la encuesta realizada por el Global Findex en 2017, hay cuatro razones principales, que hasta ese entonces derivaban en que solo el $29 \%$ de la población adulta tuviera una cuenta financiera: (i) falta de dinero, (ii) altos costos de mantener una cuenta, (iii) desconfianza en el sistema financiero, y (iv) la distancia al punto de acceso más cercano al sistema financiero.

Ahora bien, las mencionadas barreras pueden categorizarse en dos subgrupos: barreras de oferta (factores que no dependen de un individuo, sino que son características del mercado) y barreras de demanda (barreras cuya superación dependen de la propia persona).

En el caso peruano, y similar a lo que ocurre con México y Chile, el peso relativo de ambos subgrupos es similar. Más precisamente, el $84 \%$ de la gente que no tiene cuenta financiera considera su respuesta en alguna barrera de la demanda, mientras que el 75 \% señala alguna barrera del lado de la oferta (Milton y Aurazo; 2017). De este modo pueden sintetizarse los motivos que explican los bajos porcentajes de los indicadores en el caso peruano. 


\section{Análisis comparado}

Tras analizar de manera general el sector Fintech y la inclusión financiera en México, Chile y Perú, denota que existe un factor común que claramente relaciona los casos entre sí: el compromiso por la inclusión financiera y el convencimiento de las Fintech como herramienta para lograrla.

Según un estudio realizado por el Banco BBVA en el año 2017, Chile, México y Perú son de los países en la región que disponen de los mayores marcos regulativos «facilitadores» (facilitan la adopción y adaptación de las innovaciones que permiten, de forma segura, aumentar el uso de los servicios financieros por parte de grandes segmentos de la población, especialmente la población más pobre) únicamente situándose por detrás de Paraguay (Pacheco \& Rojas, 2017).

A continuación, se expone el estado de avance de las principales áreas que se creen más relevantes al momento de entender las condiciones para la inclusión financiera en cada país mediante dos figuras. La Figura 5 corresponde al Microscopio Global 2015; mientras que la Figura 6 es de la edición Microscopio Global 2018 (en este último siendo 1 un nivel bajo, 2 un nivel intermedio y 3 un nivel elevado).

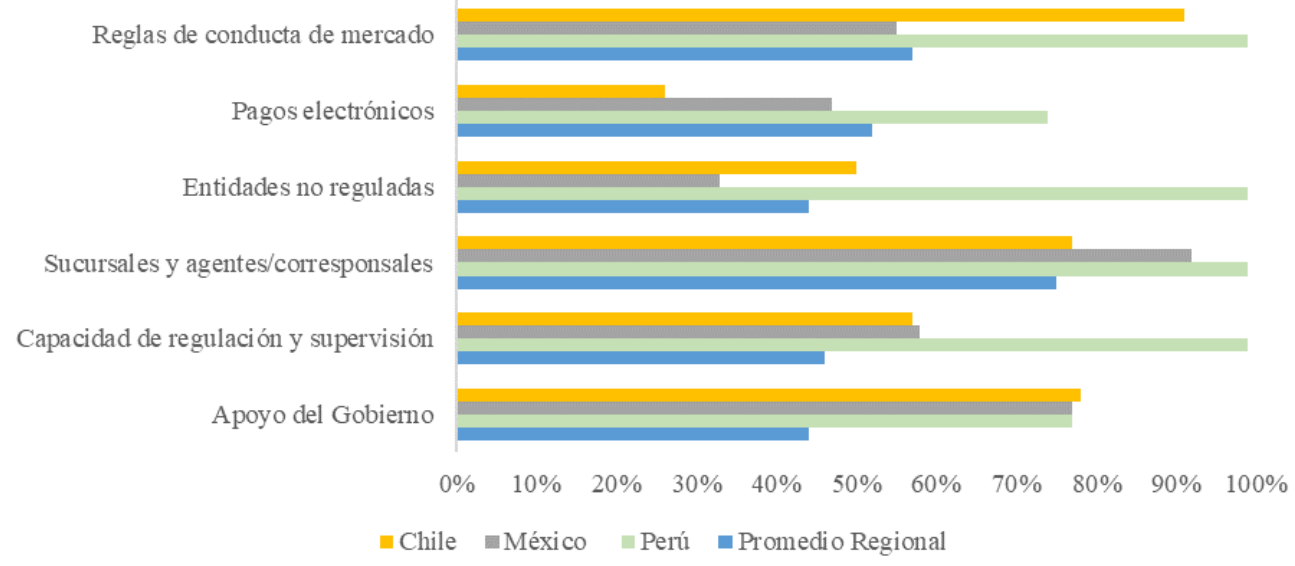

Figura 5. Estado de avance de las principales áreas relativas a la inclusión financiera para cada caso escenario Figure 5. Progress of the main aspects related to financial inclusion in each scenario Fuente: elaboración propia basada base en The Economist Intelligence Unit (2015).

Como puede observarse, con la salvedad del apoyo del Gobierno, en los restantes aspectos los países considerados se encuentran en distintas etapas de desarrollo. En paralelo, debe decirse que hay una tendencia a que el entorno para la inclusión financiera sea cada vez más favorable si se considera la evolución entre lo registrado por el estudio en 2015 contra aquello en 2018. 
Captación por parte del consumidor

Pagos electrónicos

Coordinación entre sector público y privado

Apoyo del Sector Privado

Nivel de regulación y supervisión

Apoyo del Gobierno
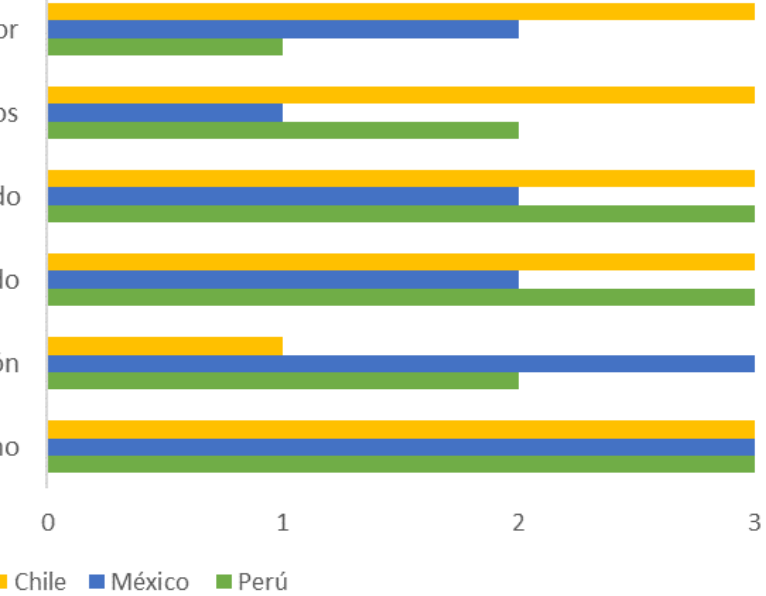

Figura 6. Estado de avance de las principales áreas relativas a la inclusión financiera para cada caso escenario

Figure 6. Progress of the main aspects related to financial inclusion in each scenario

Fuente: elaboración propia basada base en The Economist Intelligence Unit (2018).

Tal y como se muestra en la Tabla 1, las Fintech han sabido ser protagonistas en cada uno de los países, a pesar de las diferencias expuestas entre los casos. El fenómeno ha ido surgiendo en los países recibiendo el apoyo, tanto del Estado como del capital privado, y se espera que su participación en el mercado sea cada vez mayor. Solo para dimensionar una idea cercana, la consultora Ernst \& Young estima que solo en México las Fintech serán grandes competidoras en los próximos años, haciéndose del $30 \%$ del mercado financiero valuado en más de 30 mil millones de dólares (Crotte Alvarado et. al., 2018).

Tabla 1. Fortalezas y debilidades del marco regulatorio e institucional para cada caso escenario Table 1. Strengths and weaknesses of the regulatory and institutional framework in each scenario

\begin{tabular}{|c|c|c|}
\hline & Fortalezas & Debilidades \\
\hline \multirow{6}{*}{ México } & Sólidas políticas de competencia. & \multirow{2}{*}{$\begin{array}{l}\text { Ningún marco normativo en vigor permite la provisión de } \\
\text { dinero electrónico por parte de entidades no bancarias. }\end{array}$} \\
\hline & $\begin{array}{l}\text { El marco de supervisión se sustenta en amplios } \\
\text { poderes y facultades de supervisión. }\end{array}$ & \\
\hline & $\begin{array}{l}\text { Los sistemas de información crediticia abarcan } \\
\text { información exhaustiva, con altos estándares de } \\
\text { seguridad y a la que pueden acceder prestatarios y } \\
\text { prestamistas. }\end{array}$ & $\begin{array}{l}\text { Las restricciones sobre las tasas y comisiones que se } \\
\text { cobran por las cuentas simplificadas podrían dificultar el } \\
\text { diseño de modelos de negocios viables. }\end{array}$ \\
\hline & $\begin{array}{l}\text { Todos los proveedores de crédito, tanto regulados } \\
\text { como no regulados, están sujetos a las normas de } \\
\text { divulgación de información y protección de los } \\
\text { consumidores. }\end{array}$ & \multirow[t]{3}{*}{$\begin{array}{l}\text { Los procesos de supervisión para autorizar y controlar las } \\
\text { actividades de los corresponsales son complejos y exigen } \\
\text { mucho tiempo. }\end{array}$} \\
\hline & $\begin{array}{l}\text { Los bancos y otras entidades del sistema de ahorro } \\
\text { y crédito popular pueden ofrecer una amplia gama } \\
\text { de servicios a través de los corresponsales. }\end{array}$ & \\
\hline & $\begin{array}{l}\text { La intervención del estado en los mercados de } \\
\text { crédito se lleva a cabo a través de préstamos de } \\
\text { bancos de desarrollo sin que haya indicios de } \\
\text { distorsiones. }\end{array}$ & \\
\hline & Sólidas políticas de competencia. & \multirow{2}{*}{$\begin{array}{l}\text { La existencia de un impuesto que grava las operaciones } \\
\text { de crédito y los topes a las tasas de interés crea múltiples } \\
\text { distorsiones. }\end{array}$} \\
\hline Chile & $\begin{array}{l}\text { El marco de supervisión se sustenta en amplios } \\
\text { poderes y facultades de supervisión. }\end{array}$ & \\
\hline
\end{tabular}




\begin{tabular}{|c|c|c|}
\hline & $\begin{array}{l}\text { Las cuentas simplificadas ofertadas por el banco } \\
\text { público BancoEstado se aprovechan para la } \\
\text { distribución de transferencias monetarias } \\
\text { condicionadas. }\end{array}$ & $\begin{array}{l}\text { No existe un marco normativo específico para los } \\
\text { corresponsales, lo que puede generar incertidumbre } \\
\text { normativa en lo que respecta a las actividades que } \\
\text { pueden llevar a cabo estos agentes o las tasas que pueden } \\
\text { cobrar. }\end{array}$ \\
\hline & $\begin{array}{l}\text { como no regulados, están sujetos a las normas de } \\
\text { divulgación de información y protección de los } \\
\text { consumidores. }\end{array}$ & $\begin{array}{l}\text { El potencial del dinero electrónico no se aprovecha para } \\
\text { la distribución de transferencias monetarias } \\
\text { condicionadas. }\end{array}$ \\
\hline & $\begin{array}{l}\text { Prestamistas y prestatarios pueden acceder a los } \\
\text { sistemas de información crediticia con } \\
\text { disposiciones firmes para garantizar la protección } \\
\text { de los datos personales. }\end{array}$ & \\
\hline \multirow{5}{*}{ Perú } & $\begin{array}{l}\text { El marco de supervisión se sustenta en amplios } \\
\text { poderes de supervisión y disposiciones normativas } \\
\text { que garantizan la independencia del supervisor. }\end{array}$ & $\begin{array}{l}\text { La normativa en materia de cuentas simplificadas tiene } \\
\text { algunas limitaciones, como, por ejemplo, que solo están } \\
\text { disponibles para las personas físicas. }\end{array}$ \\
\hline & $\begin{array}{l}\text { Marco normativo específico sobre las cuentas } \\
\text { simplificadas que permite su distribución a través } \\
\text { de canales adecuados. }\end{array}$ & $\begin{array}{l}\text { Las medidas estatales adicionales para promover el uso } \\
\text { de las cuentas simplificadas desplazan las medidas del } \\
\text { sector privado. }\end{array}$ \\
\hline & $\begin{array}{l}\text { Las disposiciones normativas sobre el dinero } \\
\text { electrónico siguen mejores prácticas. }\end{array}$ & \multirow{3}{*}{$\begin{array}{l}\text { El potencial del dinero electrónico no se aprovecha para } \\
\text { la distribución de transferencias monetarias } \\
\text { condicionadas. }\end{array}$} \\
\hline & $\begin{array}{l}\text { Las normas relativas a los corresponsales siguen } \\
\text { mejores prácticas. }\end{array}$ & \\
\hline & $\begin{array}{l}\text { Los sistemas de información crediticia abarcan } \\
\text { información exhaustiva, con altos estándares de } \\
\text { seguridad y a la que pueden acceder prestatarios y } \\
\text { prestamistas. }\end{array}$ & \\
\hline
\end{tabular}

Fuente: elaboración propia con base en (Pacheco \& Rojas, 2017).

No solo la extensión de las Fintech ya existentes es cada vez mayor, sino que también han ido incrementando en cantidad. Entre mediados de 2018 y mediados de 2017, Perú experimento un crecimiento del $256 \%$ en cuanto a su cantidad de emprendimientos Fintech, México del $52 \%$, Chile del $29 \%$ y se espera que a nivel Latinoamérica este sea del 65 \% (BID, 2018).

En torno a la regulación, las plataformas Fintech desafían constantemente al sistema financiero tradicional con nuevos modelos de negocios innovadores y canales modernos de llegada al cliente. Al mismo tiempo, los gobiernos son conscientes del potencial que puede llegar a tener el sector Fintech sobre el desarrollo de las economías, al reducir costos operativos y al aumentar la competitividad del mercado. Como indica la Tabla 2, el marco institucional y político es vital para que prosperen los avances tecnológicos y junto a estos el funcionamiento del sector Fintech.

México ha sido el ejemplo pionero sobre cómo regular el sector Fintech de una manera holística y con proporcionalidad. Los primeros avances del proyecto se dieron en julio de 2016, para finalmente ser aprobado por la Cámara de Diputados el 1 de marzo de 2018, convirtiéndose así en la primer Ley Fintech en la región. El objetivo de esta ley fue ofrecer una mayor certeza jurídica mediante un marco legislativo que regule las plataformas denominadas Instituciones de Tecnología Financiera, además de promover un marco de competencia justa entre las Fintech y el sistema financiero tradicional (BID, 2018). 
Tabla 2. Marco regulatorio sobre las Fintech en cada caso escenario Table 2. Regulatory framework for Fintech in each scenario

\begin{tabular}{|c|c|c|c|}
\hline & México & Chile & Perú \\
\hline Tendencia regulatoria & Regulación particular & - & Regulación particular \\
\hline Marco Legal & $\begin{array}{l}\text { Anteproyecto de Ley de Tecnología } \\
\text { Financiera o Ley Fintech }\end{array}$ & - & $\begin{array}{l}\text { Proyecto de Ley que regula las } \\
\text { Plataformas de } \\
\text { Financiamiento Participativo } \\
\text { de Valores o PFPV }\end{array}$ \\
\hline $\begin{array}{l}\text { Plataformas } \\
\text { tecnológicas que } \\
\text { regula/busca regular }\end{array}$ & $\begin{array}{l}\text { Instituciones de Tecnología Financiera } \\
\text { (ITF): créditos, intercambio de activos } \\
\text { virtuales, administración de fondos de } \\
\text { pago electrónico, otros servicios } \\
\text { financieros. }\end{array}$ & - & Crowdfunding Equity \\
\hline $\begin{array}{l}\text { Requisitos de la } \\
\text { plataforma }\end{array}$ & $\begin{array}{l}\text {-SA o SRL - Actividades reguladas, } \\
\text { idoneidad de directivos, infraestructura y } \\
\text { controles internos. }\end{array}$ & - & $\begin{array}{l}\text { SA con objeto exclusivo - } \\
\text { Autorización de la SMV. }\end{array}$ \\
\hline Domicilio & México & - & Perú \\
\hline Capital mínimo & $\begin{array}{l}\text { Establecido por la CNBV en función al tipo } \\
\text { de actividad y riesgo. }\end{array}$ & - & $\begin{array}{l}\text { A ser fijado por SMV, } \\
\text { íntegramente aportado y } \\
\text { pagado en efectivo. }\end{array}$ \\
\hline $\begin{array}{l}\text { Deberes y obligaciones } \\
\text { de la plataforma }\end{array}$ & $\begin{array}{l}\text { Segregar recursos propios/clientes e } \\
\text { identificados por cada cliente (fideicomisos } \\
\text { de administración o cuentas de depósitos } \\
\text { condicionados) -Contar con EEFF } \\
\text { auditados. }\end{array}$ & - & $\begin{array}{l}\text { Informar riesgos de inversión } \\
\text { Anticipar que no se encuentra } \\
\text { supervisada por la SMV o SBS }\end{array}$ \\
\hline Prohibiciones & Asegurar retornos o rendimientos & - & $\begin{array}{l}\text { Asegurar captación de fondos } \\
\text { o garantizar a inversionistas } \\
\text { retorno o devolución }\end{array}$ \\
\hline
\end{tabular}

En el caso de Chile, no hay leyes o regulaciones específicas sobre el sector por lo que las iniciativas Fintech han tenido que ir adaptándose al marco regulatorio del sistema financiero tradicional. De todos modos, desde hace unos años las autoridades económicas del país evalúan la posibilidad de generar un marco normativo específico, particularmente para la actividad de crowfunding, que ha tenido un mayor desarrollo relativo a nivel nacional. Esta iniciativa busca generar una regulación clara, que resuelva los conflictos potenciales entre la regulación financiera general y ciertas modalidades de la operación Fintech (SBIF, 2016).

Por último, el caso peruano cuadra como un intermedio entre los dos ya mencionados. Por el momento, la Superintendencia de Mercado de Valores únicamente ha elaborado un proyecto que regula las plataformas de financiamiento participativo. Las cláusulas y requisitos de este ya fueron mencionadas, con el claro objetivo de proteger los intereses de los inversores. La Asociación Fintech Perú también quiere hacer extensiva esta regulación tanto sobre el Financiamiento Participativo a través de valores, como también a través de préstamos (Campos \& Huangal, 2017). 


\section{CONCLUSIONES}

En este trabajo se ha estudiado el fenómeno reciente de las Fintech, en cuanto a su relación con la inclusión financiera. Específicamente, se han abordado estos fenómenos en tres contextos particulares de América Latina: los países de México, Chile y Perú.

Como se ha evidenciado en cada caso, además de la estructura institucional, se pudo estudiar cuáles han sido los tipos de Fintech a los que mayor protagonismo se les ha dado. Adicionalmente, se estudió de manera descriptiva-cuantitativa la evolución reciente del fenómeno de inclusión financiera.

Para ello fue necesario brindar al menos dos definiciones conceptuales en calidad de marco teórico: inclusión financiera y Fintech, pero también fue necesario dedicar algunas líneas al sector de las microfinanzas. Como se detalló, más allá del reciente auge de la inclusión financiera, las microfinanzas han estado realizando inclusión financiera dirigida a los más vulnerables desde mediados de la década de 1970.

Seguidamente, se realizó el análisis de tres casos de estudio seleccionados para América Latina. Los corolarios expuestos son muchos y variados. Principalmente, con base en este análisis preliminar, podemos afirmar que el camino hacia la inclusión financiera digital no es uno solo. Las tres economías seleccionadas han denotado una participación público-privada muy diferenciada y, aun así, alcanzado importantes logros en materia de inclusión financiera y desarrollo del sector Fintech.

Este análisis comparado permite concluir que el fenómeno de digitalización de las finanzas, que ha sido adoptado por los promotores de la inclusión financiera, no puede ser algo que intente estudiarse de manera general. Por el contrario, las particularidades y las aristas propias en cada mercado a analizar merecen ser estudiadas en profundidad para poder diagramar un mapa más certero de oportunidades y desafíos.

Con este análisis preliminar se ha reflexionado sobre la importancia que tienen las Fintech en la actualidad y se deja una hoja de ruta a estudiar en futuras investigaciones sobre los distintos caminos a adoptar para fomentar el desarrollo tecnológico con vistas a promover la inclusión financiera.

\section{REFERENCIAS}

Aguilar, M. R. (2016). La agenda 2030 de Desarrollo Sostenible: una oportunidad para el cambio que nos compromete. Temas para el debate, (254), 22-25. Recuperado de https://dialnet.unirioja.es/servlet/articulo?codigo $=5336544$

Alliance for Financial Inclusion, AFI. (2016). The 2016 Maya Declaration Report. Recuperado de https://www.afi-global.org/publications/2359/The-2016-Maya-Declaration-Report

Arner, D. W., Barberis, J., \& Buckey, R. P. (2017). Fintech, Regtech, and the reconceptualization of financial regulation. Northwestern Journal of International Law \& Business, 37(3), 370-413. Recuperado

de https://heinonline.org/HOL/LandingPage?handle=hein.journals/nwjilb37\&div=17\&id=\&page= 
Asociación de Bancos del Perú, ASBANC. (2017). Una mirada al fenómeno Fintech en Perú y en el mundo. Departamento de Estudios Económicos. Recuperado de https://www.asbanc.com.pe/publicaciones/asbanc-semanal-242.pdf

Banco Estado (2015). Reporte de sostenibilidad 2015. Recuperado de https://www.corporativo.bancoestado.cl/sites/default/files/documentos archivos/reportesostenibilidad-2015-bancoestado-microempresas.pdf

Banco Interamericano de Desarrollo, BID (2018). Fintech: América Latina 2018: Crecimiento y consolidación. Recuperado de https://publications.iadb.org/es/Fintech-america-latina-2018crecimiento-y-consolidacion

Bermeo-Giraldo, M. C., Álvarez-Agudelo, L., Ospina-Rúa, M. I., Acevedo-Correa, Y., \& MontoyaRestrepo, I. A. (2019). Factores que influyen en la intención de uso de las tarjetas de crédito por parte de los jóvenes universitarios. Revista CEA, 5(9), 77-96. https://doi.org/10.22430/24223182.1257

Bosch-Liarte, J., \& Bosch-Liarte, J. (2016). Radiografía del Fintech: clasificación, recopilación y análisis de las principales startups (Tesis de maestría). Recuperado de https://upcommons.upc.edu/handle/2117/97361

Camacho-Beas, L. E. (2010). Impacto de las microfinanzas en el bienestar e importancia de las pymes en la economía mundial. Ingeniería Industrial, (28), 61-83. Recuperado de http://revistas.ulima.edu.pe/index.php/Ingenieria industrial/article/view/240

Campos, Y., \& Huangal, L. (2017). Necesidad de crear un marco regulatorio especifico para las Fintech en el Perú. (Tesis de maestría). Recuperado de http://repositorio.esan.edu.pe/handle/ESAN/1186

Carballo, I. E. (2018). Financial Inclusion in Latin America. In: Farazmand A. (eds.) Global Encyclopedia of Public Administration, Public Policy, and Governance. Springer, Cham. https://doi.org/10.1007/978-3-319-31816-5

Chishti, S., Barberis, J., \& Vidal, M. (2017). El futuro es Fintech. Barcelona: Ediciones Deusto.

Comisión Nacional Bancaria y de Valores, CNBV (2017). Reporte Nacional de Inclusión Financiera. Recuperado de https://www.cnbv.gob.mx/Inclusi \%C3 \%B3n/Documents/Reportes \%20de \%20IF/Reporte \%20de \%20Inclusion \%20Financiera \%208.pdf

Consejo Nacional de Inclusión Financiera, CONAIF (2016). Política Nacional de Inclusión Financiera. Recuperado de https://www.gob.mx/cnbv/acciones-y-programas/politica-nacional-deinclusion-financiera-43631

Crotte-Alvarado, A., Rasteletti, A., Ibarrarán, P., Azuara-Herrera, O., Lagarda, G., Kaplan, D. S., et al. (2018). BID economics México: Políticas para el crecimiento inclusivo y desarrollo de la economía nacional. http://dx.doi.org/10.18235/0001238 
Demirguc-Kunt, A., Klapper, L., Singer, D., Ansar, S., \& Hess, J. (2018). The Global Findex database 2017: Measuring Financial Inclusion and the Fintech Revolution. The World Bank. https://doi.org/10.1596/978-1-4648-1259-0

Dib, D., Ramírez, J. \& Alvarado, G. (2017). Panorama del Fintech en México. Endeavor México, Recuperado de http://www.crowdfundingmexico.mx/uploads/8/7/7/2/87720184/panorama fintech mexico $\underline{\mathrm{pdf}}$

Furche, P., Madeira, C., Marcel, M., \& Medel, C. A. (2017). Fintech y la banca central en la encrucijada. Estudios Públicos, (148), 39-78. Recuperado de http://www.estudiospublicos.cl/index.php/epublicos/article/view/2

G20 Research Group (2010). Principles for Innovative Financial Inclusion. Recuperado de http://www.g20.utoronto.ca/2010/to-principles.html

GPFI (2016). Global Standard-Setting Bodies and Financial Inclusion: The Evolving Landscape. Recuperado de https://www.gpfi.org/sites/default/files/documents/GPFI_WhitePaper_Mar2016.pdf

Lacalle-Calderón (2008). Microcréditos y pobreza: de un sueño al Nobel de la Paz. Recuperado de https://bit.ly/2WTISOq

Maldonado, J. H., Benitez Duarte, A. A., Gómez Soto, F., Villada V., I., Navarrete Cano, J. J., Moreno Sánchez, R. P., Bird, M., Romero Posada, M., et al. (2018). Proyecto capital: diez años vinculando la Inclusión financiera con la protección social. Recuperado de http://repositorioproyectocapital.com/wp-content/uploads/2018/08/libro-proyecto-capitalvinculando-inclusion-social-2018.pdf

Manyika, J., Lund, S., Singer, M., White, O., \& Berry, C. (2016). Digital finance for all: Powering inclusive growth in emerging economies. Mckinsey Global Institute. Recuperado de https://mck.co/2kYzyYo

Milton, V., \& Aurazo, J. (2017). ¿Por qué no tienes una cuenta? Identificando barreras a la inclusión financiera en el Perú. Revista Moneda, (170), 21-25. Recuperado de https://ideas.repec.org/a/rbp/moneda/moneda-170-04.html

Ministerio de Desarrollo Social (2015). Inclusión Financiera en Chile El desafío de diseñar una política para incluir a los grupos más vulnerables. Chile. Recuperado de https://docplayer.es/67065445-Inclusion-financiera-en-chile.html

Ocampo, M. (2017). Fintech: Tecnología Financiera. Recuperado de https://www.foroconsultivo.org.mx/INCYTU/documentos/Completa/INCYTU_17-006.pdf 
Ontiveros, E., Enríquez, Á. M., \& López, V. (2014). Microfinanzas y TIC: Experiencias innovadoras en Latinoamérica. Recuperado de https://www.findevgateway.org/es/library/microfinanzas-ytic-experiencias-innovadoras-en-latinoam \%C3 \%A9rica

Orozco-Gutierrez, M. (2019). El microcrédito, elemento clave del desarrollo económico rural: un estudio de caso. Revista CEA, 5(9), 147-159. https://doi.org/10.22430/24223182.1313

Raccanello, K., \& Herrera, E. (2014). Educación e inclusión financiera. Revista Latinoamericana de Estudios Educativos, 44(2). Recuperado de https://www.redalyc.org/html/270/27031268005/

Rojas, L. (2016). N 24. La revolución de las empresas Fintech y el futuro de la Banca. Disrupción tecnológica en el sector financiero. Caracas: CAF. Recuperado de http://scioteca.caf.com/handle/123456789/976

Rojas-Suárez, L. \& Pacheco, L. (2017). Índice de prácticas regulatorias para la Inclusión Financiera en América Latina: Facilitadores, Promotores y Obstaculizadores. Recuperado de https://www.bbvaresearch.com/wp-content/uploads/2017/08/Practicas-regulatorias-para-lainclusion-financiera-en-America-Latina-2017.pdf

Silva, A., \& Ramos, M. (2017). La evolución del Sector Fintech, modelos de negocio, regulación y retos. Documento de coyuntura 2017-02. Recuperado de https://bit.ly/2IUjHYY

Superintendencia de Bancos e Instituciones Financieras Chile, SBIF. (2016). Informe de Inclusión Financiera. Chile. Recuperado de https://www.sbif.cl/sbifweb/servlet/Publicaciones?indice=15.3\&idPublicacion=557\&idConten ido=12462\&idCategoria $=2501$

The Economist Intelligence Unit (2015). Global microscope 2015: el entorno propicio para la inclusión financiera y la expansión de los servicios financieros digitales. Recuperado de http://graphics.eiu.com//assets/images/public/Microscope_on_Microfinance_2014/EIUMicroscope-Dec-2015.pdf

The Economist Intelligence Unit (2018). Microscopio global 2018: Análisis del entorno para la inclusión financiera y la expansión de los servicios financieros digitales. Recuperado de https://www.findevgateway.org/sites/default/files/publication files/es microscopio 2018.p $\underline{\mathrm{df}}$

Torres, R. (2017). Incorporación de las nuevas tecnologías al negocio bancario en España: impacto de las Fintech. Recuperado de http://repositorio.upct.es/bitstream/handle/10317/6008/tfg-torinc. pdf? sequence $=1 \&$ isAllowed $=y$

Trivelli, C., y Caballero, E. (2018). ¿Cerrando brechas?: las estrategias nacionales de inclusión financiera en América Latina y el Caribe. Recuperado de http://repositorio.iep.org.pe/handle/IEP/1132 
Fintech e inclusión financiera: los casos de México, Chile y Perú

Varas, R. (2017). Fintech Vs el Sistema Financiero Tradicional ¿Cuál es su destino? ¿Colaboración o rivalidad? Recuperado de https://www.entrepreneur.com/article/280279

Vázquez, L. (2016). Las TIC como herramienta para la mejora del servicio a usuarios de microfinanzas. Revista Observatorio de la Economía Latinoamericana, 1-20. Recuperado de https://www.centrumthink.pucp.edu.pe/Docs/files/fintech vs sistema financiero tradiciona If.pdf

Vega, J. A. (2017). Diagnóstico y propuestas de mejoras del nivel de inclusión financiera en Chile (Tesis de pregrado). Recuperado de https://repositorio.usm.cl/handle/11673/24089

Villacorta, O., \& Reyes, J. D. (2012). Servicios financieros para las mayorías. La inclusión financiera en México. Revista de Microfinanzas y Banca Social, 2, 5-21. Recuperado de https://www.publicacionescajamar.es/pdf/publicaciones-periodicas/revista-demicrofinanzas-y-banca-social-mbs/2/2-556.pdf

World Bank Group. (2017). Global Financial Development Report 2017/2018: Bankers without Borders. Recuperado de https://www.worldbank.org/en/publication/gfdr

Zamalloa, J. C., Obando Peralta, E. C. \& Rodríguez Cairo, V. (2016). Inclusión financiera en distritos de Perú: Enfoque multidimensional y factores determinantes. Quipukamayoc, 24(46), 111-126. https://doi.org/10.15381/quipu.v24i46.13246

Ziegler, T., Reedy, E. J., Le, A., Zhang, B., Kroszner, R. S., \& Garvey, K. (2017). The Americas Alternative Finance Industry Report. Recuperado de https://bit.ly/2JMq9iM 